\title{
DDPM-DEM Simulations of Particulate Flows in Human Tracheobronchial Airways
}

\author{
Yu Feng \\ Department of Mechanical Engineering \\ North Carolina State University \\ Raleigh, NC, USA \\ yfeng4@ncsu.edu
}

\author{
Clement Kleinstreuer \\ Department of Mechanical Engineering \\ North Carolina State University \\ Raleigh, NC, USA \\ ck@ncsu.edu
}

\begin{abstract}
Dense particle-suspension flows in which particle-particle interactions are a dominant feature encompass a diverse range of industrial and geophysical contexts, e.g., slurry pipeline, fluidized beds, debris flows, sediment transport, etc. The one-way dispersed phase model (DPM), i.e., the conventional one-way coupling EulerLagrange method is not suitable for dense fluid-particle flows [1]. The reason is that such commercial CFD-software does not consider the contact between the fluid, particles and wall surfaces with respect to particle inertia and material properties. Hence, two-way coupling of the Dense Dispersed Phase Model (DDPM) combined with the Discrete Element Method (DEM) has been introduced into the commercial CFD software via in-house codes. As a result, more comprehensive and robust computational models based on the DDPMDEM method have been developed, which can accurately predict the dynamics of dense particle suspensions.

Focusing on the interaction forces between particles and the combination of discrete and continuum phases, inhaled aerosol transport and deposition in the idealized tracheobronchial airways [2] was simulated and analyzed, generating more physical insight. In addition, it allows for comparisons between different numerical methods, i.e., the classical one-way Euler-Lagrange method, two-way Euler-Lagrange method, EL-ER method [3], and the present DDPMDEM method, considering micron- and nano-particle transport and deposition in human lungs.
\end{abstract}

\section{GEOMETRY AND MESH}

A representative triple bifurcation bronchial airway model was selected to test the suitability of the DDPM-DEM method (see Fig. 1). The dimensions of the triple bifurcating geometry are for adults with a lung volume of $3500 \mathrm{~mL}$. To represent bifurcating airways starting from different generations, the triple bifurcating airway geometry was scaled to duplicate the hydraulic diameter $\mathrm{D}_{1}$ of the first bifurcation (see Fig. 1(a)) of different generations. For example, $D_{1}=0.6 \mathrm{~cm}$ represents the G3-G6 bifurcating lung airways, while $D_{1}=0.026 \mathrm{~cm}$ represents the G6-G9 bifurcating lung airways.

For the numerical simulation a structured, multi-block, body-fitted hexahedral mesh was developed (see Fig. 1(b)). Mesh independence tests have been successfully executed and presented in published papers [3]. The final mesh contained 628,712 cells, 655,822 nodes, and $1,912,466$ faces.

\section{DDPM-DEM GOVERNING EQUATIONS}

For dense particle-suspension transport and particle deposition in lung airway bifurcations it is assumed that the airflow field is laminar and incompressible. The transient 3-D DDPM-DEM method is executed as follows.

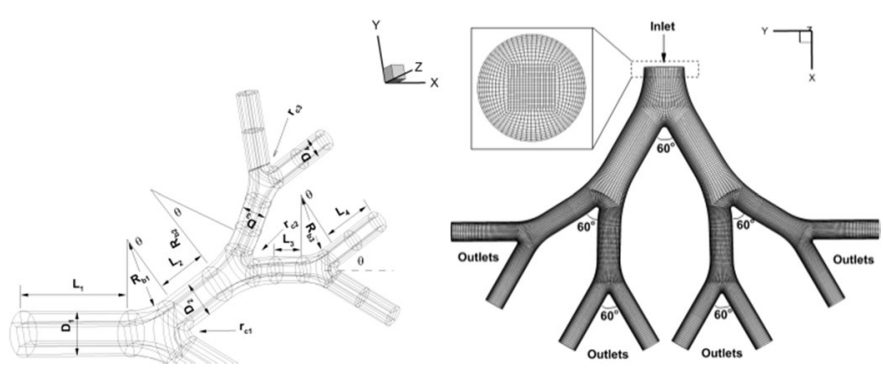

(a)

(b)

Fig. 1: (a) Configuration of Weibel's triple bifurcating airway geometry (b) Finite volume mesh of Weibel's triple bifurcating lung airway geometry

DDPM Governing Equations for Continuous Phase:

$$
\begin{gathered}
\frac{\partial\left(\alpha_{f} \rho_{f}\right)}{\partial t}+\nabla \cdot\left(\alpha_{f} \rho_{f} \overrightarrow{v_{f}}\right)=0 \\
\frac{\partial\left(\rho_{f} \alpha_{f} \overrightarrow{v_{f}}\right)}{\partial t}+\nabla \cdot\left(\alpha_{f} \rho_{f} \overrightarrow{v_{f}} \cdot \overrightarrow{v_{f}}\right)=-\alpha_{f} \nabla p+\nabla \cdot\left(\alpha_{f} \vec{\tau}_{f}\right)+\alpha_{f} \rho_{f} \vec{g}-\overrightarrow{R_{s l}}
\end{gathered}
$$

Here, $\alpha_{f}$ is the porosity, $\vec{\tau}_{f}$ is the local stress tensor, and ${\overrightarrow{R_{s l}}}_{\text {is }}$ the volumetric fluid-particle interaction force which can be expressed as:

$$
\overrightarrow{R_{s l}}=\left(\sum_{i=1}^{k_{V}} \vec{F}_{D, i}\right) / \Delta V
$$

where index $\mathrm{k}_{\mathrm{v}}$ is the number of particles in the specific mesh cell, $\Delta \mathrm{V}$ is the volume of the current mesh cell, and $\mathrm{F}_{\mathrm{D}, \mathrm{i}}$ is the drag force.

\section{DEM Translational Equation for Discrete Phase:}

Due to the relatively small dimension of, say, micron particles, only the translational equation for the dense particulate discrete phase was considered, i.e.,

$$
m_{p, i} \frac{d \vec{v}_{p, i}}{d t}=\sum_{j}\left(\vec{F}_{c n, i j}+\vec{F}_{d n, i j}+\vec{F}_{c, i j}^{t}\right)+\vec{F}_{D, i}+\vec{F}_{g, i}
$$

The normal contact force $\vec{F}_{c n, i j}$, normal damping force $\vec{F}_{d n, i j}$, tangential contact force $\vec{F}_{c, i j}^{t}$, and Stokes drag force $\vec{F}_{D, i}$ are:

$$
\begin{gathered}
\vec{F}_{c n, i j}=-k_{n} \delta_{n i j}^{3 / 2} \vec{n}_{i j} \\
\vec{F}_{d n, i j}=-\eta_{n i j} \vec{v}_{p n, i j}
\end{gathered}
$$




$$
\begin{gathered}
\vec{F}_{c, i j}^{t}=\mu_{f r i c t i o n}\left|\vec{F}_{c, i j}^{n} \vec{v}_{p t, i j} /\right| \vec{v}_{p t, i j} \mid \\
\vec{F}_{D, i}=\left(\pi d_{p, i}^{2} / 8\right) C_{D} \rho_{f}\left(\vec{v}_{p, i}-\vec{v}_{f}\right)\left|\vec{v}_{p, i}-\vec{v}_{f}\right|
\end{gathered}
$$

The details for the force calculations are given in [3].

\section{NUMERICAL SET-UP}

The governing equations, subject to appropriate boundary conditions, were numerically solved using Fluent 14.0 enhanced by inhouse user-defined functions (UDFs). To guarantee there is no inlet airflow effect, a fully-developed parabolic velocity profile was assumed. The averaged inlet Reynolds number was either 1000 or 2000. A random-parabolic distribution of micron particles at the inlet was invoked. Pressure outlet boundary conditions were employed.

\section{RESULTS AND DISCUSSION}

Comparison between DDPM-DEM and DPM:

As compared to experimental data, Figs. 2 (a) and (b) show that the combined DDPM-

DEM accurately predicts the transport and deposition of dilute particle suspensions (here, less than $1.85 \%$ ) in the G3-G6 bifurcating lung airways. Additionally, because the DDPM-DEM takes into account particle-particle interactions, it will be more accurate in simulating severe conditions, such as large pressure differentials, high velocity gradients, and intense particle collisions. It should be noted that the DPM results by Chen et al. [4] are somewhat off for various reasons, as discussed by Feng [3].

Deposition Patterns:

Figures 3(a) and 3(b) depict deposited micron particles
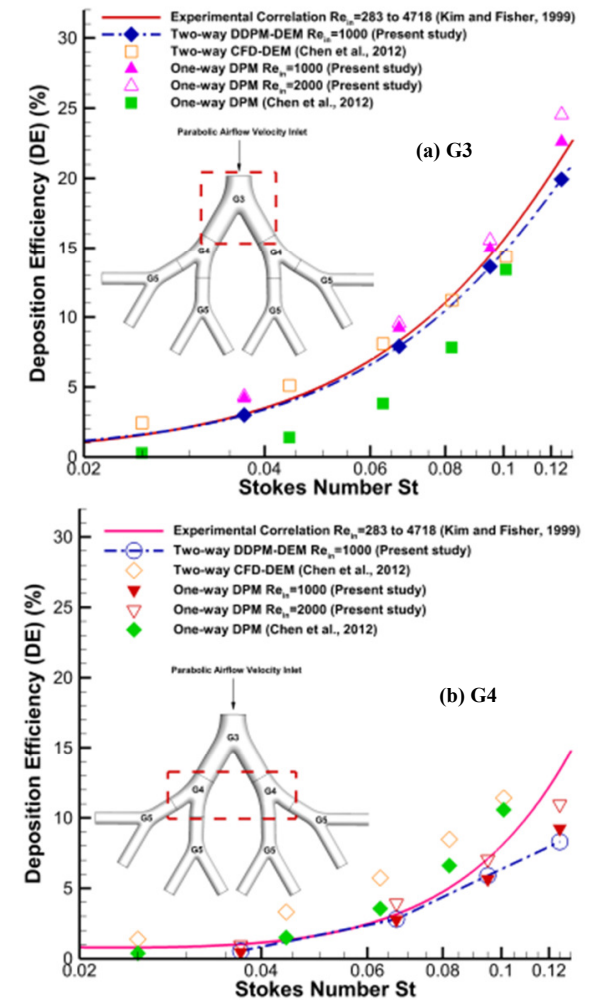

Fig. 2: Comparison between computational data using different numerical methods and measured particle deposition efficiency data correlation [1] for the first

$\left(\mathrm{d}_{\mathrm{p}}=10 \mu \mathrm{m}\right)$ at $\mathrm{G} 3$ to $\mathrm{G} 6$ for different Stokes numbers. Particle deposition occurs at the stagnation points due to direct impaction and gravitational sedimentation. The asymmetric particle deposition patterns are due to the particle-particle contact interaction, and random particle inlet distribution. Clearly, higher Stokes numbers induce a stronger impaction effect on particle deposition.

\section{Transport Characteristics:}

Particle transport patterns at different time steps are shown in Figs. 4(a) to 4(f). The particles obtained similar velocity distributions during the air-solid coupling process. The impaction and particle splash near the first bifurcation can be observed clearly from Figs. 4(c) and 4(d).

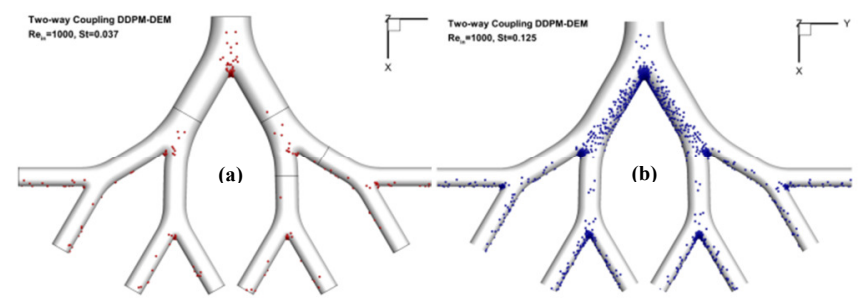

Fig. 3: Particle $\left(d_{p}=10 \mu \mathrm{m}\right)$ deposition comparisons in bifurcating airway model G3-G6 using two-way coupling DDPM-DEM (a) $\mathrm{St}=0.037$ (b) $\mathrm{St}=0.125$

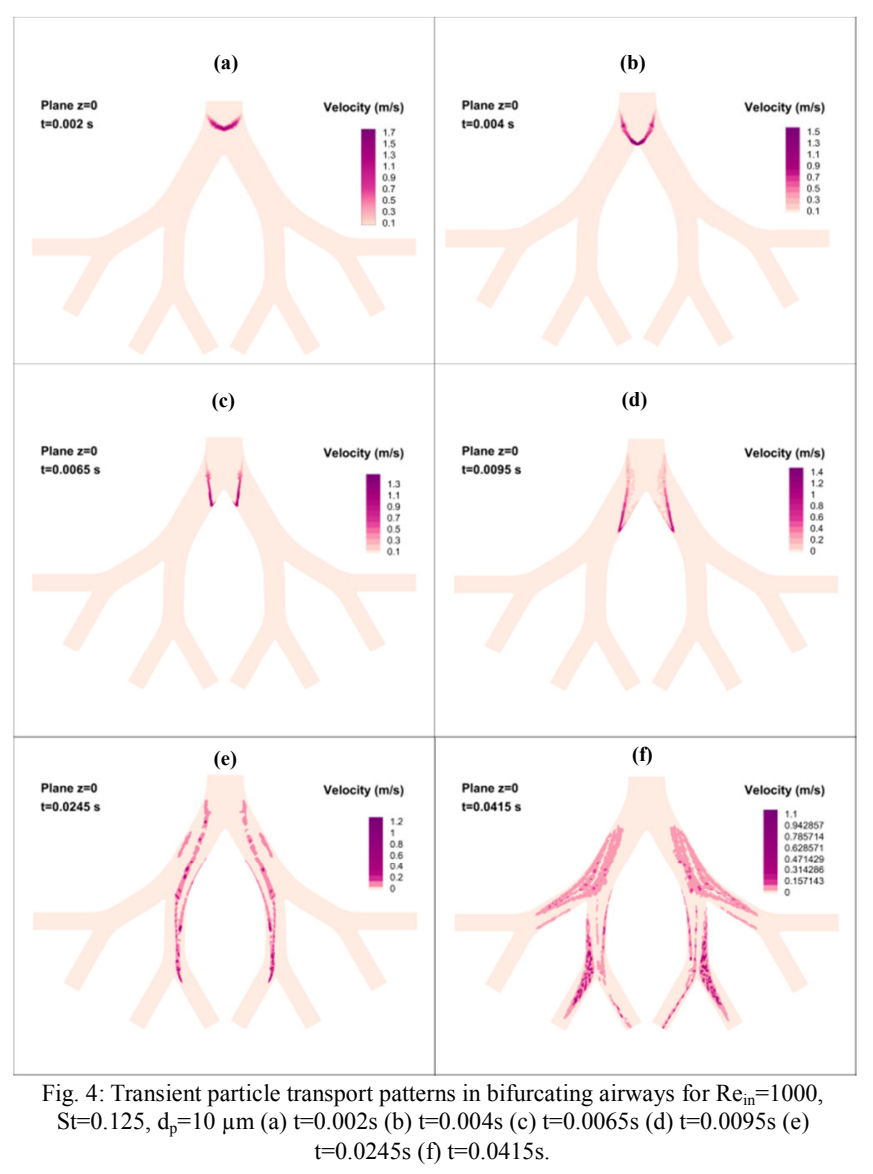

\section{ACKNOWLEDGEMENTS}

The authors are grateful for the financial support of Y. F. by a grant from NSF (CBET-1232988), Environmental Health and Safety of Nanotechnology, Dr. Barbara Karn, Program Director.

\section{REFERENCES}

[1] Crowe, C. T., Schwarzkopf, J. D., Sommerfeld, M., Tsuji, Y., 2011, "Multiphase Flows with Droplets and Particles", 2nd edition, Boca Raton, FL, USA: CRC Press.

[2] Kim, C. S., and Fisher, D. M., 1999, "Deposition Characteristics of Aerosol Particles in Sequentially Bifurcating Airway Models", Aerosol Science \& Technology, 31(2-3), pp. 198-220

[3] Feng, Y., 2013, "Computational Ellipsoidal Particle-Fluid Analysis and Discrete Element Method with Applications to Particle Transport and Deposition in Human Respiratory Models", Ph. D. Dissertation, NC State University, Raleigh, NC, USA

[4] Chen, X., Zhong, W., Zhou, X., Jin, B., \& Sun, B. (2012). CFDDEM simulation of particle transport and deposition in pulmonary airway. Powder Technology, 228, pp. 309-318 